\title{
A putative new SARS-CoV protein, 3c, encoded in an ORF overlapping ORF3a
}

\author{
Andrew E. Firth*
}

\begin{abstract}
Identification of the full complement of genes in severe acute respiratory syndrome coronavirus 2 (SARS-CoV-2) is a crucial step towards gaining a fuller understanding of its molecular biology. However, short and/or overlapping genes can be difficult to detect using conventional computational approaches, whereas high-throughput experimental approaches - such as ribosome profiling - cannot distinguish translation of functional peptides from regulatory translation or translational noise. By studying regions showing enhanced conservation at synonymous sites in alignments of SARS-CoV-2 and related viruses (subgenus Sarbecovirus) and correlating the results with the conserved presence of an open reading frame (ORF) and a plausible translation mechanism, a putative new gene - ORF3c - was identified. ORF3c overlaps ORF3a in an alternative reading frame. A recently published ribosome profiling study confirmed that ORF3c is indeed translated during infection. ORF3c is conserved across the subgenus Sarbecovirus, and encodes a 40-41 amino acid predicted transmembrane protein.
\end{abstract}

\section{FINDINGS}

The aetiological agent of coronavirus disease 2019 (COVID$19)$ is the virus severe acute respiratory syndrome coronavirus 2 (SARS-CoV-2), a coronavirus in the genus Betacoronavirus, subgenus Sarbecovirus. Like other coronaviruses, SARSCoV-2 has a positive-sense RNA genome that is approximately $30000 \mathrm{nt}$ in size. The $5^{\prime}$ two-thirds of the genome contain two long open reading frames (ORFs), ORF1a and ORF1b, which are translated from the viral genomic RNA (gRNA). ORFs 1a and $1 \mathrm{~b}$ encode the polyproteins ppla and pplab, where translation of pplab depends on a proportion of ribosomes making a programmed $-1 \mathrm{nt}$ ribosomal frameshift near the end of ORF1a to enter ORF1b. Polyproteins ppla and pplab are proteolytically processed to produce the viral replication proteins (reviewed in [1]). The $3^{\prime}$ third of the genome contains a number of ORFs that encode the viral structural and accessory proteins. These ORFs are translated from a nested series of subgenomic mRNAs (sgmRNAs) that are produced during the infection cycle (reviewed in [2]). In SARS-CoV-2, these ORFs comprise S, 3a, E, M, 6, 7a, 7b, $8, \mathrm{~N}, 9 \mathrm{~b}$ and possibly 10 (Fig. 1a). The S, E, M and N ORFs encode respectively the virus spike, envelope, membrane and nucleocapsid proteins - key components of the virus particle that are conserved among coronaviruses. ORFs 3a, 6, 7a, $7 \mathrm{~b}, 8$ and $9 \mathrm{~b}$ encode accessory proteins, also present in the
2002-2003 severe acute respiratory syndrome coronavirus (hereafter SARS-CoV-1 to avoid any confusion), but less widely conserved across coronaviruses as a whole (reviewed in [3]). These 3'-proximal ORFs each have a corresponding dedicated sgmRNA, except for ORFs $7 b, 9 b$ and 10 . ORFs $7 \mathrm{~b}$ and $9 \mathrm{~b}$ appear to be translated from the ORF7a and $\mathrm{N}$ sgmRNAs, respectively, via a leaky scanning mechanism $[4,5]$ whereas the translation mechanism for ORF10 is unknown.

There is considerable variability between coronavirus genera and subgenera in the complement of 3 '-encoded accessory genes [3]. Even within the sarbecovirus subgenus, there are differences. For example, SARS-CoV-1 has an ORF3b that overlaps the $3^{\prime}$ region of ORF3a but is truncated or absent in SARS-CoV-2. Also, in many human-adapted SARS-CoV-1 isolates, ORF8 is split by a frame-disrupting deletion [6]. ORF10 is apparently translated in SARS-CoV-2 [7] but is truncated in SARS-CoV-1. Identification of the full complement of genes in SARS-CoV-2 is a crucial step towards gaining a fuller understanding of its molecular biology, and may also help guide vaccine or other antiviral strategies. This information also facilitates rational manipulation of the viral genome (e.g. for developing replicon systems or for mutagenesis studies). However, short and/or overlapping genes can be particularly difficult to identify using

Received 13 May 2020; Accepted 25 June 2020; Published 15 July 2020

Author affiliations: 'Division of Virology, Department of Pathology, Addenbrooke's Hospital, University of Cambridge, Cambridge, UK.

*Correspondence: Andrew E. Firth, aef24@cam.ac.uk

Keywords: SARS-CoV; coronavirus; overlapping gene; ORF3c; 3c; sarbecovirus.

Abbreviations: COVID-19, coronavirus disease 2019; gRNA, genomic RNA; ORF, open reading frame; SARS-CoV, severe acute respiratory syndrome

coronavirus; sgmRNA, subgenomic mRNA.

001469 (c) 2020 The Authors 


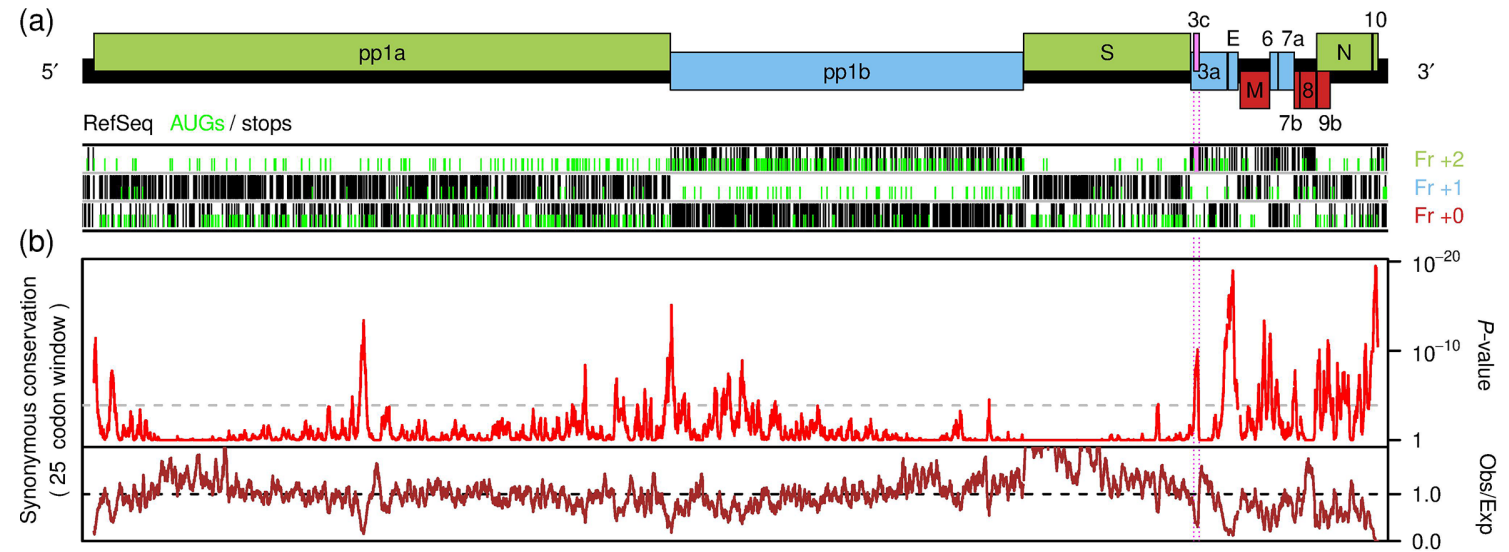

\begin{abstract}
Fig. 1. Synonymous site conservation analysis of sarbecoviruses. (a) Map of the SARS-CoV-2 genome (29903 nt; black rectangle). Known ORFs are overlaid in red, blue or green depending on their relative reading frames $(+0,+1,+2$, respectively). Below are shown the positions of AUG (green) and stop (black) codons in each of the three reading frames, as indicated, in the reference sequence NC_045512.2. The putative ORF3c is indicated in pink (reading frame +2). (b) Synonymous site conservation analysis of 54 aligned sarbecovirus sequences. The red line shows the probability that the observed conservation could occur under a null model of neutral evolution at synonymous sites, whereas the brown line depicts the ratio of the observed number of substitutions to the number expected under the null model. The horizontal dashed grey line indicates a $P=0.05$ threshold after an approximate correction for multiple testing, namely scaling by ( 25 codon window size)/(length of plot in codons). Prior to analysis, the alignment was mapped to NC_045512.2 coordinates by removing alignment positions in which NC_045512.2 contained a gap character. NCBI accession numbers: NC_045512.2, AY274119.3, DQ022305.2, DQ071615.1, DQ084199.1, DQ084200.1, DQ412042.1, DQ412043.1, DQ648856.1, DQ648857.1, GQ153539.1, GQ153540.1, GQ153541.1, GQ153542.1, GQ153543.1, GQ153544.1, GQ153545.1, GQ153546.1, GQ153547.1, GQ153548.1, GU190215.1, JX993987.1, JX993988.1, KC881006.1, KF294457.1, KF367457.1, KF569996.1, KJ473811.1, KJ473812.1, KJ473813.1, KJ473816.1, KP886808.1, KP886809.1, KT444582.1, KY352407.1, KY417142.1, KY417143.1, KY417145.1, KY417146.1, KY417147.1, KY417148.1, KY417149.1, KY417150.1, KY417151.1, KY417152.1, KY770858.1, KY770859.1, KY770860.1, MG772933.1, MG772934.1, MK211374.1, MK211376.1, MK211377.1 and MK211378.1.
\end{abstract}

traditional computational approaches. On the other hand, high-throughput experimental techniques such as ribosome profiling and high resolution mass spectrometry - while powerful - do not necessarily distinguish between functional proteins, regulatory translation (where it is the act of translation rather than the encoded product that is biologically relevant) and translational noise.

Comparative genomics offers a way forward: analysis of patterns of substitutions across alignments of related sequences can be used to reveal the signatures of 'hidden' protein-coding genes. Analysis of synonymous substitution rates provides a particularly sensitive technique for identifying overlapping functional elements embedded within protein-coding genes, because such elements constrain synonymous changes that are otherwise selectively more-or-less neutral [8]. When combined with the conserved presence of an ORF and the conserved presence of a plausible translation mechanism, overlapping genes may be distinguishable from overlapping non-coding elements such as functionally important RNA structures [9-12].

Sequences with $100 \%$ coverage of, and $\geq 70 \%$ amino acid identity to, the SARS-CoV-2 pplab sequence were identified with NCBI TBLASTN [13] on 12 January 2020 and downloaded. These cut-off thresholds corresponded precisely to the subgenus Sarbecovirus. Sequences that did not cover the entire protein-coding region of the genome, and all sequences with $>99 \%$ amino acid identity in pplab to SARSCoV-1 except a single reference sequence NC_004718.3 were removed. This left 54 sequences (SARS-CoV-1, SARS-CoV-2 and 52 bat coronaviruses). Codon-respecting alignments were produced using a previously described procedure [8]. In brief, each individual genome sequence was aligned to the SARS-CoV-2 reference sequence (GenBank accession number NC_045512.2) using code2aln version 1.2 [14]. Genomes were then mapped to NC_045512.2 coordinates by removing alignment positions that contained a gap character in the reference sequence, and these pairwise alignments were combined to give the multiple sequence alignment. To assess conservation at synonymous sites, the known virus coding regions were extracted from the alignment (with codons selected from the longer ORF in each overlap region) and concatenated in-frame, and the alignment was analysed with synplot2 [8] using a 25 codon sliding window. Conservation statistics were then mapped back to NC_045512.2 coordinates for plotting (Fig. 1b).

The synonymous site conservation analysis revealed conserved features towards the $5^{\prime}$ end of ORFla, in the middle of ORF1a, near the end of ORF1a and start of ORF1b, and in many parts of the $3^{\prime}$ region of the viral genome. Most of these conserved elements do not correspond to conserved overlapping ORFs and likely represent functional RNA sequence elements - a case in point being the frameshift-stimulating 


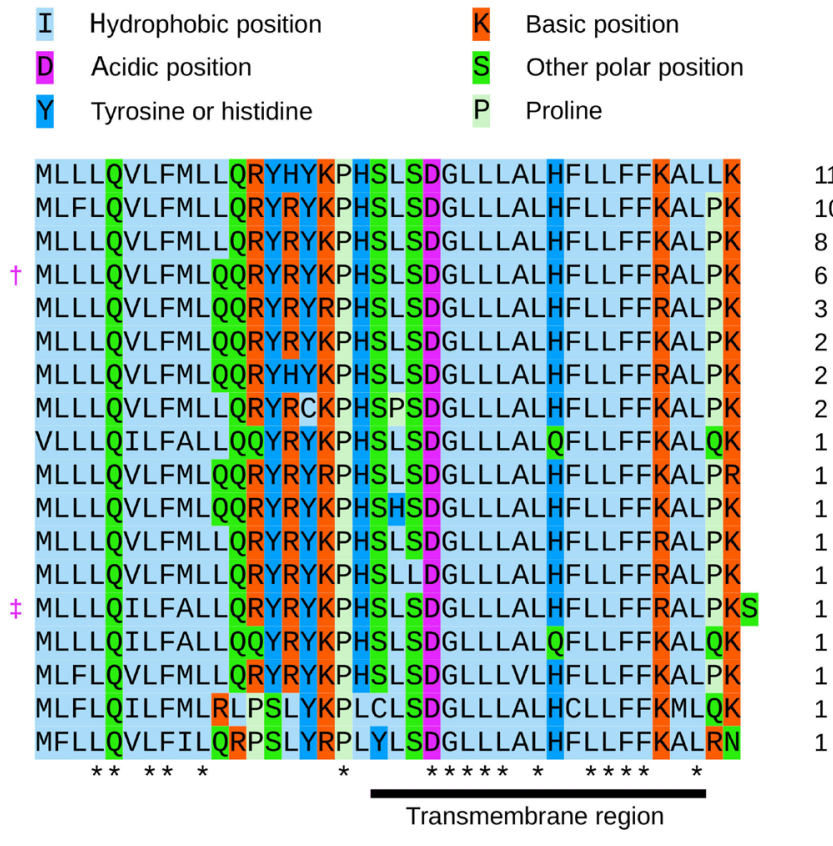

Fig. 2. Amino acid alignment of sarbecovirus $3 c$ sequences. Amino acids are colour-coded according to their physicochemical properties. Asterisks indicate completely conserved columns in the alignment. The transmembrane region predicted by Phobius is indicated with a black bar below the alignment. Numbers at the right indicate the number of times the particular sequence occurs among the 54 sarbecovirus sequences (see Fig. 1 caption for accession numbers). †, SARS-CoV-1. $\neq$, SARS-CoV-2. For the sequence beginning with GUG instead of AUG, the genetic decoding (i.e. valine) is shown, even though non-AUG initiation codons are normally expected to be decoded as methionine by initiator Met-tRNA.

RNA pseudoknot [15] at the junction of ORFs $1 \mathrm{a}$ and $1 \mathrm{~b}$. However, a peak in conservation within ORF3a stood out because, although short, it coincides with an overlapping alternative-frame AUG-initiated ORF - hereafter ORF3c positioned close to the $5^{\prime}$ end of ORF3a, where it might be accessible via ribosomal leaky scanning (reviewed in [16]).

Closer inspection revealed that the presence and location of the $3 c$ initiation and stop codons were conserved across sarbecoviruses. The $3 \mathrm{c}$ AUG codon is present in all but 1 of the 54 sequences, where it is replaced with GUG (MG772933, a bat coronavirus). In all 54 sequences, there is an $\mathrm{A}$ at the -3 position, giving a strong initiation context [17]. The GUG in MG772933 may also serve as an initiation site, as GUG codons can be utilized for initiation - at a reduced efficiency compared to AUG - when in a strong initiation context (reviewed in [16]). In 52 sequences there are 2 upstream AUGs - the ORF3a AUG (intermediate context; $\mathrm{C}$ or $\mathrm{U}$ at -3 and $G$ at +4 ) and another AUG also in the ORF3a reading frame (weak context; $\mathrm{U}$ at -3 and $\mathrm{A}$ at +4 ); in the remaining two sequences the second of these AUGs is absent. The lack of a strong initiation context for either of the upstream AUG codons might enable a proportion of preinitiation scanning $43 \mathrm{~S}$ complexes loaded on the ORF3a sgmRNA to leaky scan to the $3 c$ initiation codon. The $3 c$ ORF has a length of 40 codons in all 54 sequences except 1, namely SARS-CoV-2, where it is 41 codons in length (Fig. 2). The SARS-CoV-2 putative $3 \mathrm{c}$ protein has a molecular mass of $4.9 \mathrm{kDa}$ and a pI of 10.9. The protein is also predicted to contain a transmembrane domain (via Phobius [18]) (Fig. 2). Curiously, the transmembrane amino acids are relatively highly conserved, suggesting that they may form interactions within the lipid bilayer, for example, membrane-disrupting or membraneassociated signalling activities.

Ribosome profiling is a high-throughput sequencing technique that allows footprinting of initiating and/or elongating ribosomes at sub-codon resolution and hence the global identification of initiation sites and/or sequence regions and reading frames undergoing translation $[19,20]$. A recent ribosome profiling study of cells infected with SARS-CoV-2 revealed 23 novel translated ORFs [7]. Ten are very short $(\leq 15$ codons). Seven of the remainder comprise $5^{\prime}$ extensions or $5^{\prime}$ truncations of previously known ORFs (M, 6, 7a, 7b, 9b and 10). Two are uORFs positioned on the gRNA upstream of ORF1a that may play a role in regulating ORF1a/1b expression as previously proposed for uORFs in other coronaviruses [19, 21]. After excluding these ORFs, only four novel translated ORFs remain: ORF3c (25457-25582; 41 codons), another ORF overlapping ORF3a (25596-25697; 33 codons), an ORF overlapping the S ORF (2174421863; 39 codons) and a truncated version of the same (21768-21863; 31 codons) (where numbers indicate ORF coordinates in NC_045512.2). To investigate the four overlapping ORFs, the ORF S and ORF 3 a regions were extracted from all 54 sarbecovirus sequences, translated to amino acids and aligned using MUSCLE [22], and the amino acid alignments were used to guide codonrespecting nucleotide sequence alignments (EMBOSS tranalign [23]). These alignments were analysed with synplot2, again using a 25 codon window size. Of the four translated novel ORFs (Fig. 3, yellow rectangles), only ORF3c was found to coincide with a synonymous site conservation signal. Moreover, the other three novel ORFs are not conserved: in many sarbecovirus sequences they lack the AUG codon and are interrupted by stop codons (Fig. 3).

Although synonymous site conservation can result from overlapping non-coding or coding elements, the conserved presence and conserved positions of the ORF $3 \mathrm{c}$ start and stop codons suggests the latter interpretation. Moreover, the ribosome profiling study of Finkel et al. [7] confirms that ORF3c is indeed translated during infection. The combination of comparative genomics showing purifying selection (which to a large extent is synonymous with functional importance) and ribosome profiling showing expression strongly suggests that $3 \mathrm{c}$ is a functional protein, conserved throughout sarbecoviruses. While the known SARS-CoV-2 genes have already been investigated in SARS-CoV-1 (reviewed in [3]), $3 c$ has never before been studied. Clearly, additional work with SARS-CoV reverse genetics systems will be required to elucidate the $3 c$ protein function, and it may eventually provide a new target for vaccine or antiviral strategies. The synplot2 analysis (Fig. 1b) has also revealed other functional 

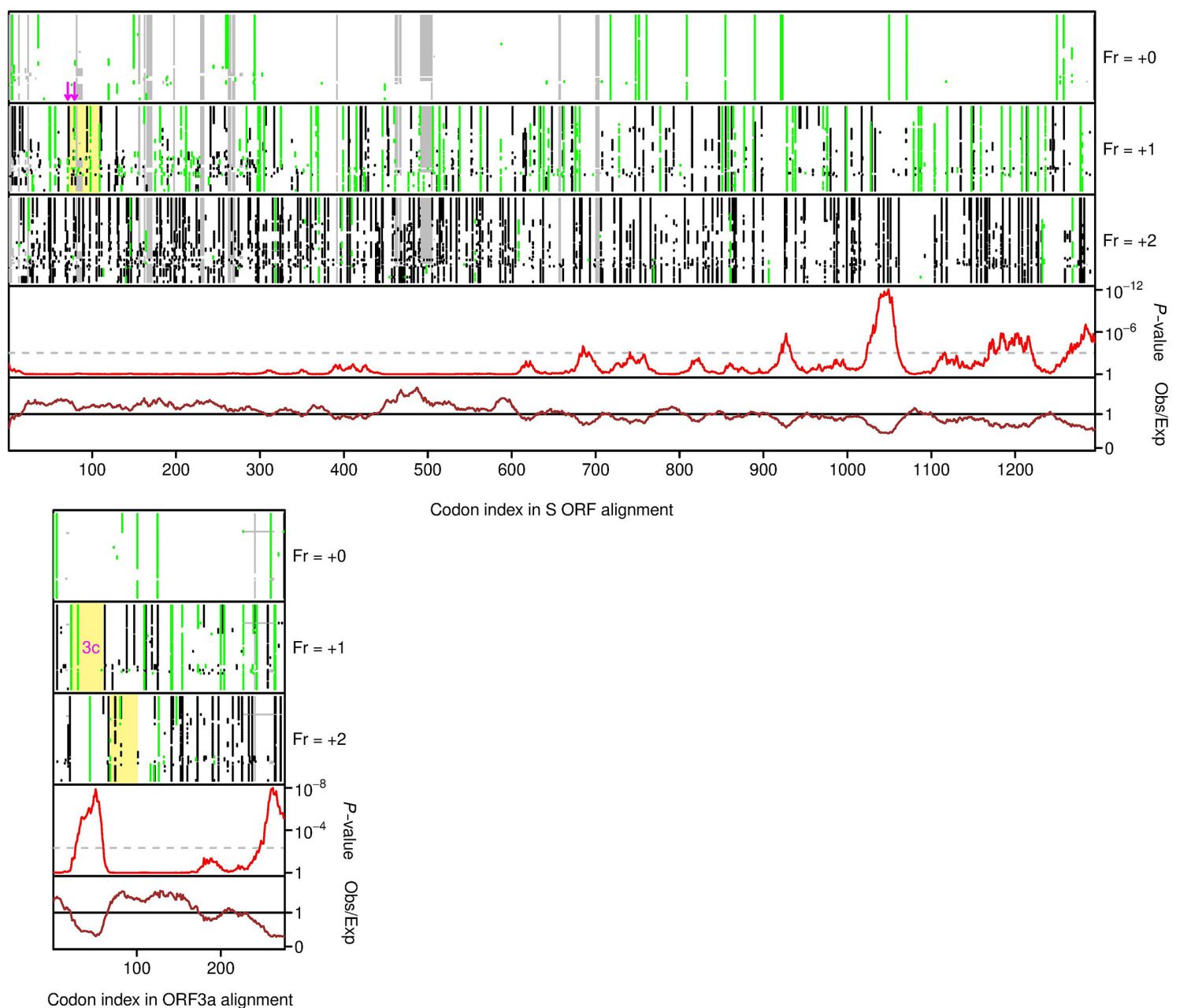

Fig. 3. Conservation analyses of the sarbecovirus $S$ and 3a ORFs. In each plot, the upper three panels show the positions of alignment gaps (grey), stop codons (black) and AUG codons (green) in each reading frame in each of the 54 aligned sequences. In these plots the canonical ORFs (i.e. S and 3a) are taken as reading frame +0. Below is shown the analysis of conservation at synonymous sites (see Fig. 1 caption for details). In contrast to Fig. 1, here all alignment gaps were retained instead of mapping to NC_045512.2 coordinates. Novel alternative-frame translated ORFs identified in the SARS-CoV-2 ribosome profiling study of Finkel et al. [7] are indicated with yellow rectangles; for S, the two in-frame alternative initiation sites are indicated with pink arrows. ORF3c is labelled. Note that only ORF3c has conserved start and stop codon positions across sarbecoviruses and only ORF3c coincides with a region of enhanced synonymous site conservation.

elements embedded within the viral protein-coding genes (e.g. in ORF1a), which may also be worthy of experimental investigation.

During preparation of this manuscript, $3 \mathrm{c}$ was independently discovered by Cagliani et al. [24] (where it is termed $3 \mathrm{~h}$ ), who performed a similar analysis with synplot2 but used far fewer sarbecovirus sequences, and hence achieved lower statistical significance for conserved elements. More recently, $3 \mathrm{c}$ was also independently discovered by Jungreis et al. [25] (where it is also termed 3c) using PhyloCSF, in which ORF3c-frame codon substitutions are compared with coding and non-coding evolutionary models, thus representing an independent approach that is completely different from that used in synplot 2 .

\section{Funding information}

This work was supported by a Wellcome Trust grant (106207) and a European Research Council grant (646891) to A. E. F.

\section{Acknowledgements}

I thank Hazel Stewart, Valeria Lulla, David Matthews, Edward Emmott, Betty Chung, James Edgar, Ian Brierley, Andrew Davidson and Nina Lukhovitskaya for helpful discussions.

Conflicts of interest

The author declares that there are no conflicts of interest. 
References

1. Snijder EJ, Decroly E, Ziebuhr J. The nonstructural proteins directing coronavirus RNA synthesis and processing. Adv Virus Res 2016;96:59-126

2. Sola I, Almazán F, Zúñiga S, Enjuanes L. Continuous and discontinuous RNA synthesis in coronaviruses. Annu Rev Virol 2015;2:265-288

3. Liu DX, Fung TS, Chong KK-L, Shukla A, Hilgenfeld R. Accessory proteins of SARS-CoV and other coronaviruses. Antiviral Res 2014;109:97-109.

4. Schaecher SR, Mackenzie JM, Pekosz A. The ORF7b protein of severe acute respiratory syndrome coronavirus (SARS-CoV) is expressed in virus-infected cells and incorporated into SARS-CoV particles. J Virol 2007;81:718-731.

5. Xu K, Zheng B-J, Zeng R, Lu W, Lin Y-P et al. Severe acute respiratory syndrome coronavirus accessory protein 9B is a virionassociated protein. Virology 2009;388:279-285.

6. Chinese SARS Molecular Epidemiology Consortium, He J. Molecular evolution of the SARS coronavirus during the course of the SARS epidemic in China. Science 2004;303:1666-1669

7. Finkel Y, Mizrahi O, Nachshon A, Weingarten-Gabbay S, YahalomRonen $\mathrm{Y}$ et al. The coding capacity of SARS-CoV-2. bioRxiv 2020:2020.05.07.082909

8. Firth AE. Mapping overlapping functional elements embedded within the protein-coding regions of RNA viruses. Nucleic Acids Res 2014;42:12425-12439PMID.

9. Fang Y, Treffers EE, Li Y, Tas A, Sun Z et al. Efficient -2 frameshifting by mammalian ribosomes to synthesize an additional arterivirus protein. Proc Natl Acad Sci U S A 2012;109:E2920-E2928.

10. Jagger BW, Wise HM, Kash JC, Walters K-A, Wills NM et al. An overlapping protein-coding region in influenza A virus segment 3 modulates the host response. Science 2012;337:199-204.

11. Loughran G, Firth AE, Atkins JF. Ribosomal frameshifting into an overlapping gene in the $2 \mathrm{~B}$-encoding region of the Cardiovirus genome. Proc Natl Acad Sci U S A 2011;108:E1111-E1119.

12. Lulla V, Firth AE. A hidden gene in astroviruses encodes a cell-permeabilizing protein involved in virus release. bioRxiv 2019:661579.
13. Altschul SF, Gish W, Miller W, Myers EW, Lipman DJ. Basic local alignment search tool. J Mol Biol 1990;215:403-410.

14. Stocsits RR, Hofacker IL, Fried C, Stadler PF. Multiple sequence alignments of partially coding nucleic acid sequences. BMC Bioinformatics 2005;6:160.

15. Baranov PV, Henderson CM, Anderson CB, Gesteland RF, Atkins JF et al. Programmed ribosomal frameshifting in decoding the SARSCoV genome. Virology 2005;332:498-510.

16. Firth AE, Brierley I. Non-Canonical translation in RNA viruses. J Gen Virol 2012:93:1385-1409.

17. Kozak M. Point mutations define a sequence flanking the AUG initiator codon that modulates translation by eukaryotic ribosomes. Cell 1986:44:283-292.

18. Käll L, Krogh A, Sonnhammer ELL. Advantages of combined transmembrane topology and signal peptide prediction--the Phobius web server. Nucleic Acids Res 2007;35:W429-W432.

19. Ingolia NT, Ghaemmaghami S, Newman JRS, Weissman JS Genome-Wide analysis in vivo of translation with nucleotide resolution using ribosome profiling. Science 2009;324:218-223.

20. Irigoyen N, Firth AE, Jones JD, Chung BY-W, Siddell SG et al High-Resolution analysis of coronavirus gene expression by RNA sequencing and ribosome profiling. PLoS Pathog 2016;12:e1005473.

21. Wu H-Y, Guan B-J, Su Y-P, Fan Y-H, Brian DA. Reselection of a genomic upstream open reading frame in mouse hepatitis coronavirus 5'-untranslated-region mutants. J Virol 2014;88:846-858.

22. Edgar RC. Muscle: a multiple sequence alignment method with reduced time and space complexity. BMC Bioinformatics 2004:5:113.

23. Rice P, Longden I, Bleasby A. EMBOSS: the European molecular biology open software suite. Trends Genet 2000;16:276-277.

24. Cagliani R, Forni D, Clerici M, Sironi M. Coding potential and sequence conservation of SARS-CoV-2 and related animal viruses. Infect Genet Evol 2020;83:104353.

25. Jungreis I, Sealfon R, Kellis M. Sarbecovirus comparative genomics elucidates gene content of SARS-CoV-2 and functional impact of COVID-19 pandemic mutations. bioRxiv 2020:2020.06.02.130955.

\section{Five reasons to publish your next article with a Microbiology Society journal}

1. The Microbiology Society is a not-for-profit organization.

2. We offer fast and rigorous peer review - average time to first decision is 4-6 weeks.

3. Our journals have a global readership with subscriptions held in research institutions around the world.

4. $80 \%$ of our authors rate our submission process as 'excellent' or 'very good'.

5. Your article will be published on an interactive journal platform with advanced metrics

Find out more and submit your article at microbiologyresearch.org. 